\author{
Susan Henrikson \\ Caroline E. Blane \\ Khaldoun Koujok \\ Peter J. Strouse \\ Michael A. DiPietro \\ Mitchell M. Goodsitt
}

\section{The effect of screening sonography on the positive rate of enemas for intussusception}

Received: 6 June 2002

Accepted: 14 October 2002

Published online: 12 December 2002

(C) Springer-Verlag 2002
S. Henrikson · C.E. Blane ( $\square)$

K. Koujok · P.J. Strouse - M.A. DiPietro

M.M. Goodsitt

Department of Radiology,

University of Michigan Health System,

1500 East Medical Drive F3503,

Ann Arbor, MI 48109-0252, USA

Email: cblane@umich.edu

Tel.: + 1-734-7632571

Fax: + 1-734-7649351

\begin{abstract}
Background: The referring physicians at our institution used the enema as a diagnostic test in children with suspected intussusception. Objective: To determine the change in rate of positive enema findings performed for suspected intussusception with the intervention of screening ultrasound (US).

Materials and methods: Since October 1995, 224 children (mean age years) with suspected intussusception were referred for enema examination. In January 2001, US was introduced as a screening test for intussusception. Enemas were performed for all children with positive US findings and were offered for those with negative US findings if clinical suspicion persisted.

Results: Before 2001, 184 children underwent enema with intussusception documented in $40(22 \%)$. Since January 2001, 40 children have been seen with suspected intussusception
\end{abstract}

(12/40 positive or $30 \%)$. Two directly underwent enema ( $1 / 2$ positive); 38 children underwent US. In 12 of 38 children, the US finding was positive, and an intussusception was found at enema examination in 11 of 12 . In 26 cases, the US finding was negative. Seven of the 26 children with a negative sonogram finding had an enema, which was also negative. Nineteen enemas were canceled. With a screening US, the positive rate for enemas is now $58 \%(11 / 19)$. We know of no case of intussusception missed at US. Conclusion: Screening US has decreased unnecessary enemas for clinically suspected intussusception, increasing positive findings from $22 \%$ to $58 \%$. This has in turn reduced children's exposure to radiation.

Keywords Intussusception Ultrasound · Sonography $\cdot$ Enema

\section{Introduction}

One of the earliest reports of sonographic diagnosis of intussusception appeared in the literature in 1982 [1]. The children reported in that study had atypical presentations of intussusception and an ultrasound (US) study had been ordered as a screening examination for abdominal pain [1]. The intussusception was identified as a mass with the appearance of a target, donut, or bull's eye with multiple rings of differing echogenicity.
This typical appearance has been described with pathologic correlation [2]. In 1985, two reports in the literature recommended screening US for cases of suspected intussusception. Each study had 14 patients with positive US findings and proven diagnosis of intussusception $[3,4]$. Since then several other studies have advocated the use of US in the diagnosis of intussusception $[4,5,6,7,8]$. However, at our institution, the enema was still considered the examination of choice for children with the suspected diagnosis of intussusception, 
although we have anecdotal experience through the years of an intussusception being detected initially at US.

With the expansion of the healthcare network in recent years, we noticed with concern a decreasing rate of positive enema findings for suspected intussusception in the year 2000. As a group, the pediatric radiologists decided with the help of the pediatric surgeons to introduce screening sonography in all cases. An educational program was developed to teach the US technologists how to perform a screening study for intussusception and recognize the typical target mass. Examples of intussusception on sonography were also shown for teaching purposes to the radiology residents, the pediatric residents, the staff physicians, and the pediatric radiologists. The new screening US examination was discussed with the primary referring physicians, the emergency room physicians, and pediatric surgeons. In January 2001 we introduced the program with the clear understanding of the referring physicians that if they still wanted an enema for clinical reasons, even in the face of a negative US finding, it would be performed. Initially, enemas were performed regardless of whether the US findings were negative or positive for the entire team to gain confidence. Subsequently, enemas were only performed if the US finding was positive or, in rare instances, when strong clinical suspicion persisted in spite of a negative US finding. The purpose of this study is to describe our results for the 13-month period from January 2001 to February 2002.

\section{Materials and methods}

Our Institutional Review Board approved the reporting of these data which are maintained in a Quality Assurance monitor. Medical records of children with a request for an enema to exclude the suspected diagnosis of intussusception from October 1995 to January 2002 were analyzed. Patients were identified as having a clinical suspicion of intussusception based on signs and symptoms as judged by emergency room physicians, pediatricians, or pediatric surgeons. Before January 2001, enemas were performed on all patients for diagnosis and treatment. After January 2001, patients with suspected intussusception underwent initial screening sonography. Sonography technicians performed the scans using a $7.5-\mathrm{MHz}$ transducer on the GE or ATL equipment. The entire abdomen was scanned along the course of the colon for the typical appearance of a mass from intussusception as taught in our educational sessions. A positive US finding consisted of a donut sign with a hypoechoic outer ring and an echogenic center and/or concentric rings (Fig. 1). The results were reviewed initially by residents and finally by staff pediatric radiologists. All patients with positive sonography findings had an air or barium enema for treatment. All initial six cases had an enema whether the US findings were positive (2) or negative (4). After an initial four cases with negative findings at US and enema and two positive cases confirmed by enema, enemas were no longer routinely performed on every child. The results of a negative US finding were discussed with the referring physician. The clinician then decided whether they would still proceed with an enema.

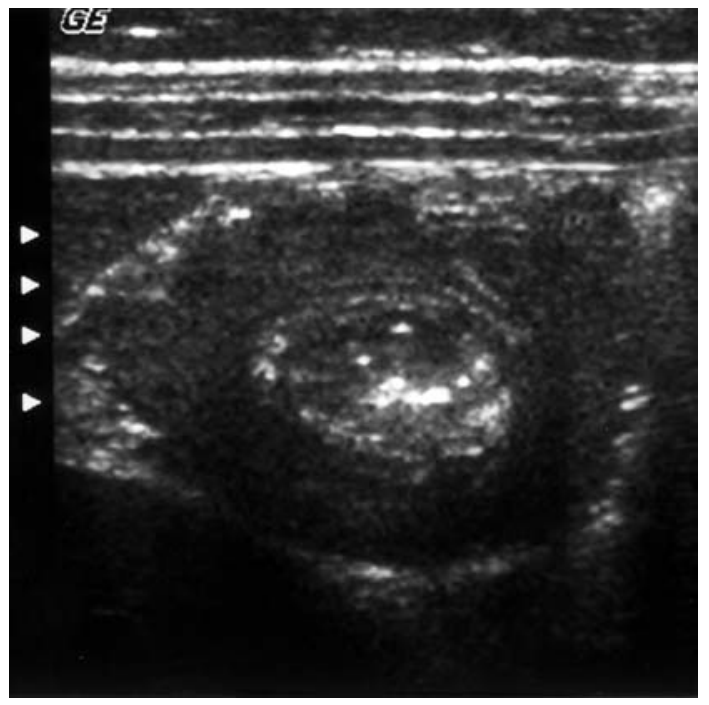

Fig. 1 Typical target lesion of intussusception at ultrasound with differing layers of echogenicity in rings

\section{Results}

Between October 1995 and December 2000 a total of 184 children were sent to our department with a request for an enema to exclude intussusception. Forty of these examinations had positive findings for a rate of $22 \%$. The rates of positive enema findings for each year were: $1996,15 \%$; 1997, 17\%; $1998,32 \% ; 1999,32 \%$; and 2000, 16\%. From January 2001 until January 2002 a total of 40 children were sent to the radiology department with the suspected diagnosis of intussusception. There were two children who went straight for an enema examination with no screening US for the suspected diagnosis of intussusception, one of which was positive and one of which was negative. This was early in the program and the resident on call at night neglected to perform the US before organizing the enema and calling the pediatric radiologist to the hospital. The initial six of 38 children, with two positive US and four negative US findings, all had an enema after the sonography. In all, 26 of 38 children had a US with negative findings with three additional enemas requested after the initial four for a total of seven enemas with negative findings. Twelve children had a US with positive findings, 11 of whom had a subsequent enema with positive findings. Repeat US after the enema in the one false-positive case at sonography showed interval resolution of the mass lesion. At the time of the enema, the ileocecal valve was noted to be prominent with evidence of mucosal edema. From this data set, 11 of 19 enemas were positive for intussusception $(58 \%)$. If we exclude the initial four negative learning/teaching US studies, the positive rate of enemas increases to $73 \%$. If only the positive cases at 
sonography were followed by enema, the positive rate would have increased to $92 \%$.

We had initially planned to continue to perform enemas on all patients for several months until staff, residents, and faculty members became comfortable with the screening US. The first six children screened with US, four with negative findings and two with positive findings, and all cases concordant on both studies convinced the referring physicians to change behavior. The referring physicians became comfortable with the results of the US much sooner than we had anticipated. In the face of a negative US finding in 22 subsequent children, 19 enemas were canceled. Excluding the two children who missed a screening US entirely, and the initial four US studies with negative findings that we insisted have an enema, there were 34 children with the suspected diagnosis of intussusception sent to radiology. There were 19 of 22 US studies with negative findings where the referring clinician canceled the request for the enema based on the results of the US. In three of the negative cases, the negative US was not sufficient evidence for the referring clinician and an enema was also requested which was negative for each of these children.

\section{Charge estimates}

We calculated the charge of introducing screening US and performing enemas in those positive or still requested cases against the charge of an enema in all cases. We used these rough numbers since collection and payer mix complicate the actual money saved. If the 38 children had directly undergone enema examination, the total charge would have been U.S. $\$ 25,308$. An enema to diagnose and, if indicated, reduce an intussusception carries a greater cost at our institution related to the setup and the presence of the radiologist on call who must come in for the procedure. The actual charges for the 38 limited abdominal US studies and the 19 enemas were U.S. $\$ 25,422$. If we calculate the charges for the 38 US studies and add 15 enemas (remove the initial four from the learning curve), the charges decrease to U.S. \$22,758. If the clinicians gain greater confidence in sonography and request enemas only in those children with a positive US, the charges would drop to U.S. $\$ 20,760$.

\section{Radiation estimates}

Computing the roughly estimated dose on four of our enemas with negative findings and four with positive findings in the group of children seen in 2001, some assumptions were made. Our fluoroscopy equipment operates at 7.5 pulses per second with image intensifier in the normal 12" mode. Abdominal exposure was estimated using linear interpolation of the doses for a 6-month-old and a 12-year-old patient, knowing the average thicknesses for children in these age groups as well the age of the individual child. The number of images, $\mathrm{kVp}$, and $\mathrm{mAs}$ was known in each case. The average radiation dose roughly calculated for negative enemas was $820 \mathrm{mR}$ (range 257-1,542 mR), and in positive enemas the average was $2,567 \mathrm{mR}$ (range $1,131-4,402 \mathrm{mR}$ ). In positive enema cases the radiation exposure to reduce the enema was also included in this calculation. Thus in each of the 19 children who had an enema canceled because the US findings were negative, the roughly calculated average radiation dose saved was $820 \mathrm{mR}$.

\section{Discussion}

The consequences of a missed intussusception can lead to significant morbidity and even mortality from ischemic bowel necrosis. Thus the referring clinician often feels compelled to order an enema even if the clinical symptoms are not classic and the plain films are unrevealing once the diagnosis has been suggested. US has been well documented as an excellent screening test for intussusception $[3,5,6,8]$. However, it has not gained wide popularity in general practice and even in some children's hospitals. We have demonstrated that a change in ordering practice by referring clinicians can be successfully introduced. We have successfully increased our positive enema rate from $22 \%$ before the introduction of screening sonography to $58 \%$ over the 13 months included in this study. If we exclude the initial learning experience, the rate of positive enemas actually increased to $73 \%$. We used conferences and dedicated teaching sessions to reach all members of the community including the clinicians, residents, sonographers, and radiologists. At the inception of the introduced change we believed that it would be 6 months before the clinicians would trust a negative US report and cancel the enema, despite the supporting literature $[3,4,5,6,7,8]$. However, in practice, the referring physicians became comfortable with the use of screening sonography very rapidly, after our initial six cases (two of which had positive and four of which had negative findings).

Although the US findings of a target lesion are not pathognomonic for intussusception, a high degree of sensitivity can be achieved with the use of US in the appropriate clinical setting $[3,5]$. We know of no child with intussusception missed at screening sonography. We had one false-positive case diagnosed as intussusception at the initial sonography with a negative enema and a negative follow-up US. We believe that this was a case of spontaneous reduction of an intussusception well documented in the literature [9]. In fact, it has been suggested that US be used to confirm reduction of the intussusception after enema in those cases where the 
swollen ileocecal valve has complicated visualization of the reduced bowel loops [9].

Patients with contraindications to enema may avoid the study entirely. Children with Henoch-Schönlein purpura (HSP) have underlying intestinal vasculitis that leads to an increased risk for perforation with enema [10]. These patients are also at increased risk for intussusception and frequently troubled with abdominal pain [10]. Sonography can be used to determine which HSP child actually has an ileocolic intussusception and would benefit from an enema or a small bowel intussusception and should proceed directly to surgical consultation [7]. The possibility of recurrent intussusception, or incompletely reduced intussusception, can be verified with sonography before proceeding with an additional enema or taking a child to surgery [5, 7]. Occasionally, alternative causes of abdominal pain such as an inflammatory process in the bowel, appendicitis, mesenteric adenitis, or twisted ovarian cyst may be identified at US.

The use of US in screening provides obvious advantages. At our institution a limited abdominal US is substantially less expensive than an enema for intussusception. In fact, doing both studies in positive cases and US alone in negative cases results in cost saving. In this day and age of increasing medical costs there are few improvements in patient care such as this one that lead to a decrease in overall charges. More importantly, we were able to eliminate the need for 19 enema examinations and the associated radiation for those 19 children. We would hope in the future to further reduce the number of enemas requested as negative US findings become accepted by the referring clinicians. Radiation of children during medical imaging has become very topical. The recent April 2002 issue of Pediatric Radiology was devoted to reporting the findings at a multidisciplinary conference on radiation associated with $\mathrm{CT}$ imaging in children. Cancellation of the 19 enemas also resulted in avoidance of an unpleasant experience of an enema on a young child with all the attendant parental anxiety and concern. In addition, since many of the children with intussusception present to the emergency room after hours, those 19 canceled enemas resulted in 19 fewer trips to the hospital by the pediatric radiologist on call.

\section{References}

1. Bowerman RA, Silver TM, Jaffe MH (1982) Real-time ultrasound diagnosis of intussusception in children. Radiology 143:527-529

2. del-Pozo G, Albillos JC, Tejedor D (1996) Intussusception: US findings with pathologic correlation. The crescent-in doughnut sign. Radiology 199:688-692

3. Swischuk LE, Hayden CK, Boulden T (1985) Intussusception: indications for ultrasonography and explanation of the doughnut and pseudokidney signs. Pediatr Radiol 15:388-391
4. Tran-Minh VA, Pracos JP, Massard PE et al (1985) Diagnosis of acute intestinal intussusception (AII) by real-time ultrasonography. Pediatr Radiol 15:267

5. Pracos JP, Tran-Minh VA, Morin De Finfe CH, et al (1987) Acute intestinal intussusception in children: contribution of ultrasonography (145 cases). Ann Radiol 30:525-530

6. Verschelden P, Filiatrault D, Garel L, et al (1992) Intussusception in children: reliability of US in diagnosis, a prospective study. Radiology 184:741-744

7. Shanbhogue RL, Hussain SM, Meradji $\mathrm{M}$, et al (1994) Ultrasonography is accurate enough for the diagnosis of intussusception. J Pediatr Surg 29:324-328
8. Bhisitkul DM, Listernick R, Shkolnik A, et al (1992) Clinical application of ultrasonography in the diagnosis of intussusception. J Pediatr 121:182-186

9. Swischuk LE, John SD, Swischuk PN, (1994) Spontaneous reduction of intussusception: verification with US. Radiology 192:269-271

10. Hu SC, Feeney MS, McNicholas M, et al (1991) Ultrasonography to diagnose and exclude intussusception in Henoch-Schönlein purpura. Arch Dis Child 66:1065-1067 